\title{
Spectroscopy of Brown Dwarf Candidates in the NGC 1333 Molecular Cloud
}

\author{
Bruce Wilking, Ayman Mikhail, and Glenn Carlson \\ Department of Physics and Astronomy, University of Missouri-St. \\ Louis, 8001 Natural Bridge Road, St. Louis, MO 63121
}

Michael R. Meyer

Steward Observatory, The University of Arizona, Tucson, AZ 85721

Thomas Greene

NASA/Ames Research Center, M.S. 245-6, Moffett Field, CA 94035

\begin{abstract}
We present an analysis of low-resolution infrared spectra for 25 brown dwarf candidates in the NGC 1333 molecular cloud. Candidates were chosen on the basis of their association with the high column density cloud core, and near-infrared fluxes and colors. We compare the depths of water vapor absorption bands in our candidate objects with a grid of dwarf, subgiant, and giant standards to determine spectral types which are independent of reddening. These data are used to derive effective temperatures and bolometric luminosities which, when combined with theoretical tracks and isochrones for pre-main sequence objects, enable us to estimate masses and ages. Depending on the models considered, a total of 9 to 20 brown dwarfs are identified with a median of age of $<1$ Myr.
\end{abstract}

\section{Introduction}

The NGC 1333 reflection nebula and its associated dark cloud L 1450 are part of a chain of molecular clouds in the Perseus region (Sargent 1979; Loren 1976). Analysis of Hipparcos data suggests a distance of 300 pc (de Zeeuw, Hoogerwerf, $\&$ de Bruijne 1999; Belikov et al. 2002). The observations of emission-line stars and Herbig-Haro objects first established NGC 1333 as an active region of star formation (e.g., Herbig 1974). Surveys of the cloud at near-infrared wavelengths have revealed a large population of low mass stars (Strom, Vrba, \& Strom 1976; Aspin, Sandell, \& Russell 1994; Lada, Alves, \& Lada 1996; Wilking et al. 2002). The young stellar objects are concentrated into a northern and southern cluster, each with about 70 members. Identification of the lowest mass objects in the NGC 1333 cloud, as well as estimates for their ages and masses, requires infrared spectroscopy. Ultimately, the population of very low mass objects will be used to define the shape of the mass function and determine the number and total mass of brown dwarfs in the young cluster. 


\section{Source Selection and Observations}

Brown dwarf candidates were selected by comparing their positions in a nearinfrared color-magnitude diagram with theoretical models for pre-main sequence objects by D'Antona \& Mazzitelli (1997, 1998, hereafter DM97). An age of 1 Myr was assumed (Lada et al. 1996). Near-infrared surveys of the northern cluster (Wilking et al. 2002) and the southern cluster (Aspin et al. 1994) were used to construct the $\mathrm{K}$ vs. (H-K) diagram. The majority of the 25 candidates lie in a region of the diagram where $\mathrm{M}<0.1 \mathrm{M}_{\odot}, \mathrm{A}_{v}<10 \mathrm{mag}$, and $\mathrm{K}<14 \mathrm{mag}$.

Infrared spectroscopic observations were accomplished using the 3-m NASA Infrared Telescope Facility at Mauna Kea, Hawaii in 2000 November 10-13. The observations were aided by an Internet2 link between UM-St. Louis and Mauna Kea, Spectra in the K band were obtained for 25 brown dwarf candidates, eight $M$ giants, and eight $M$ subgiants using the $256 \times 256$ InSb facility infrared camera (NSFCAM) with the HKL grism and a $0.3^{\prime \prime}$ pixel $^{-1}$ scale. The $0.6^{\prime \prime}$ slit provided a resolution of $\mathrm{R}=\lambda / \Delta \lambda \sim 300$ over the $2.0 \mu \mathrm{m}-2.5 \mu \mathrm{m}$ band.

\section{Results}

\subsection{Spectral Classification Using a Water Vapor Index}

In the K-band, broad water vapor absorption bands centered at $1.9 \mu \mathrm{m}$ and 2.5 $\mu \mathrm{m}$ provide the best means for deriving spectral types for faint $\mathrm{M}$ stars. These bands are not only sensitive to temperature for cool stars (Jones et al. 1994) but also are well-resolved in low resolution spectra. Water vapor indices have been used to derive spectral types for very low mass young stars in the $\rho$ Ophiuchi cloud (Wilking, Greene, \& Meyer 1999, hereafter WGM99; Cushing, Tokunaga, \& Kobayashi 2000), IC 348 (Najita, Teide, \& Carr 2000), the Orion Nebula cluster (Lucas et al. 2001), and the Taurus cloud (Itoh, Tamura, \& Tokunaga 2002). In WGM99, we defined a $Q$ index that was independent of reddening as

$$
Q=(F 1 / F 2)(F 3 / F 2)^{1.22}
$$

where F1, F2, and F3 are the average fluxes in narrow bands covering 2.07-2.13 $\mu \mathrm{m}, 2.2695-2.2875 \mu \mathrm{m}$, and $2.40-2.50 \mu \mathrm{m}$, respectively.

A linear fit to a plot of the $\mathrm{Q}$ index vs. the optically-determined $\mathrm{M}$ spectral type for dwarf standards is shown in Fig. 1 for stars from M0.5V-M9V and yields the following relation

$$
M V \text { subclass }=(-20.15 \pm 0.48) \times Q+(18.49 \pm 0.55)
$$

with a correlation coefficient of $\mathrm{r}=0.98$ for a sample of 12 measurements.

\subsection{The Water Index as a Function of Surface Gravity}

A sample of eight $M$ subgiants were selected for infrared spectroscopic observations from the MBM 12 and IC 348 young stellar populations. The median ages of these populations are estimated to be 2 Myr. As shown in Fig. 1, the linear fit to the subgiant sample is consistent with the dwarf relation and there is no apparent sensitivity of the $\mathrm{Q}$ index to surface gravities in the dwarf to subgiant 
Water Index vs. M Spectral Type

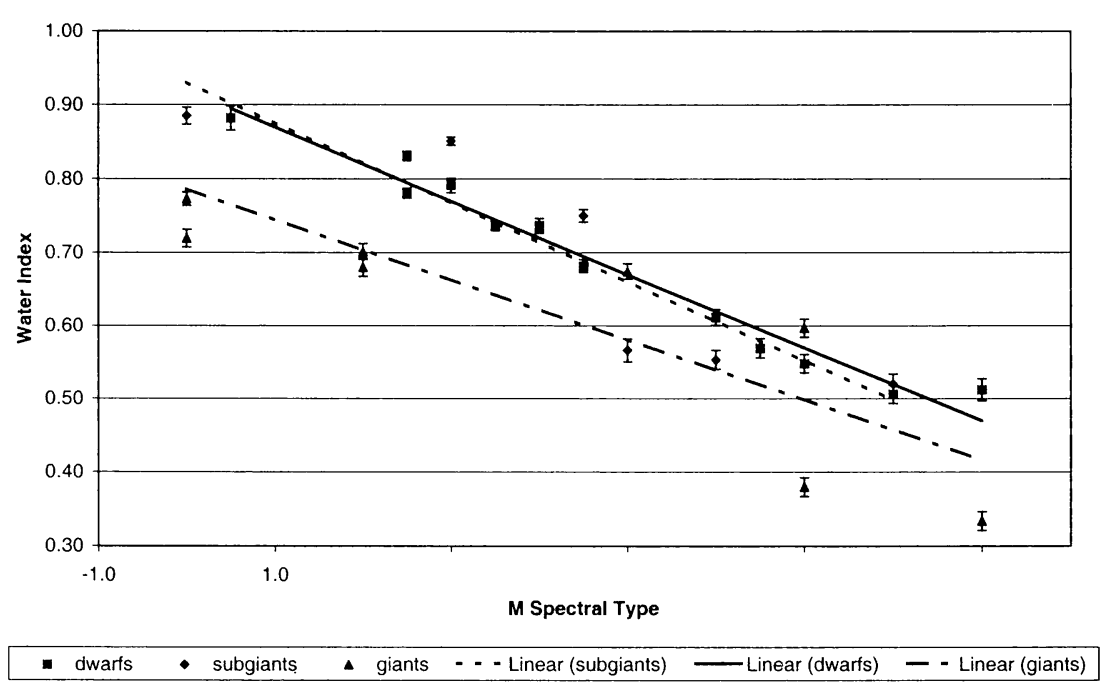

Figure 1. The $\mathrm{Q}$ index vs. $\mathrm{M}$ spectral type for dwarf standards (squares), subgiants (diamonds), and giants (triangles). Linear fits to the dwarf and subgiant data are nearly identical.

range. This is in agreement with the findings of Jones et al. (1995) who, in comparing infrared synthetic spectra, saw little dependence on the depths of the water lines with surface gravity between $\log (\mathrm{g})=4.0$ to 5.0 . The $\mathrm{Q}$ values for the 8 giants observed show a much greater scatter from a linear fit. In our sample, dwarfs always display deeper water vapor absorption than giants for a given spectral type. However, the Q index for giants can be smaller than that of dwarfs due to enhanced absorption by $\mathrm{CO}$ in the $2.40-2.50 \mu \mathrm{m}$ band. Hence, the $\mathrm{Q}$ index is not a useful measure of water vapor absorption for objects with the surface gravities of giant stars.

\section{3. $\quad$ Spectral Types}

Twenty-four brown dwarf candidates displayed late-type photospheres with absorption due to water vapor and CO characteristic of high surface gravity. Since the surface gravities of objects in this sample are likely to resemble those of subgiants, spectral types were derived using Eqn. 2 which is appropriate for both subgiants and dwarfs. Spectral classifications using the water vapor index relation reveals spectral types between M2.4 and M8.3 with typical uncertainties of \pm 0.5 subclass. Seventeen of 24 candidates displayed spectral types $\geq$ M6.0. The visual extinctions implied by these spectral types are typically 2-3 mag and all less than $10 \mathrm{mag}$. The majority of the candidates appear to be young objects of very low mass. The projection of this sample on the high column density core $\left(\mathrm{A}_{v}>10 \mathrm{mag}\right)$ coupled with the relatively low visual extinctions of the candidates minimizes the chance that some objects are background $M$ stars. The detection of x-ray emission from 15 of the 24 candidates by Getman 

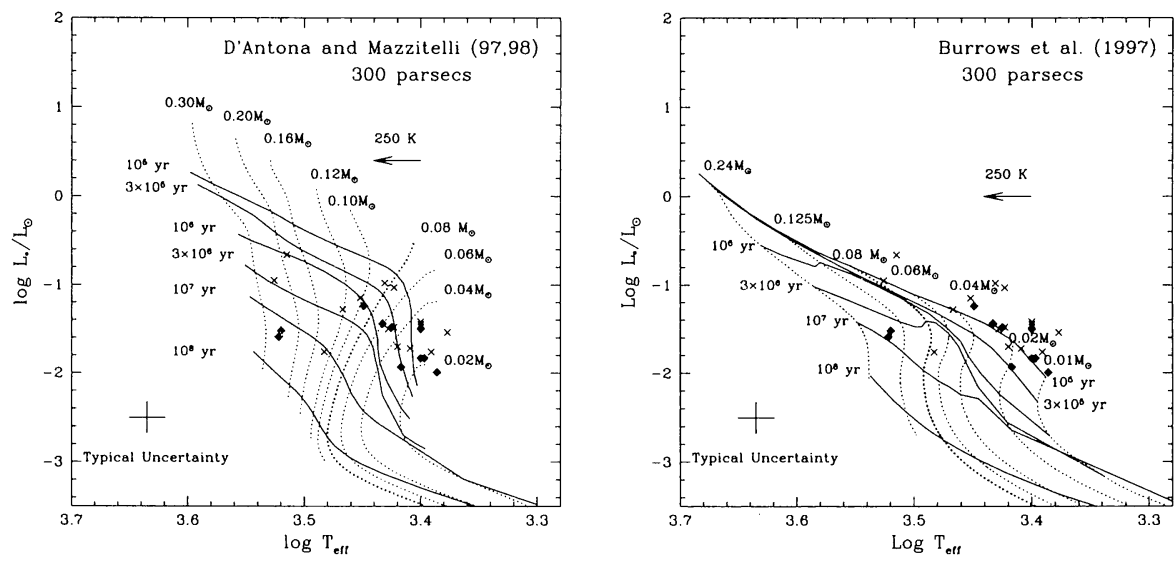

Figure 2. H-R diagrams for the brown dwarf candidates using the DM97 models (left panel) and the Burrows et al. models (right panel). ' $X$ 's mark the positions of $\mathrm{x}$-ray emitting candidates.

et al. (2002), including 3 tentative detections, argues in favor of the youth of this sample, as X-ray luminosity and stellar activity are known to decline with age for K and M stars (Fleming, Schmitt, \& Giampapa 1995).

\subsection{Masses and Ages of the Brown Dwarf Candidates}

We followed the procedure outlined in WGM99 to estimate the masses and ages of the brown dwarf candidates. Effective temperatures computed for each brown dwarf candidate have typical uncertainties of $\pm 95 \mathrm{~K}$. However, systematic effects could affect the derived temperatures. The presence of moderate veiling in 3 objects could lead us to overestimate their effective temperatures by $\sim 150$ $\mathrm{K}$. The assumption of dwarf, rather than subgiant, surface gravities could also systematically affect our results. For spectral types of M2 and later, giant star temperatures are warmer than dwarfs by $300-500 \mathrm{~K}$ for stars of the same spectral type (e.g., Fig. 4 in Itoh et al. 2002). Hence by assuming a dwarf surface gravity, we may be underestimating the effective temperature of an M2 subgiant by 150 $\mathrm{K}$ and of an M6-M9 subgiant by $\sim 250 \mathrm{~K}$.

In Fig. 2, we have plotted our brown dwarf candidates on HertzsprungRussell diagrams overlaid with the theoretical tracks and isochrones from the models of of DM97 and Burrows et al. (1997). An H-R diagram using the Baraffe et al. (1998) models is presented in Wilking et al. (2002). For the DM97 models, mass estimates range from $<0.02 \mathrm{M}_{\odot}$ to $0.25 \mathrm{M}_{\odot}$, with 16 objects at or below the hydrogen-burning limit. The median age for the sample is $0.3 \mathrm{Myr}$. If a temperature scale intermediate between dwarfs and giants is used, then these objects should be shifted to higher temperatures by $250 \mathrm{~K}$ and only 9 would remain as brown dwarfs. The Burrows et al. models yield lower masses and younger ages with 23 objects at or below the hydrogen-burning limit and 20 of these remaining as brown dwarfs if shifted to higher temperatures by $250 \mathrm{~K}$. The median age for the sample implied by the Burrows et al. models is $0.1 \mathrm{Myr}$. 


\subsection{Surface Gravity Estimates}

Surface gravities $\left(\mathrm{g}=\mathrm{GM} / \mathrm{R}^{2}\right)$ can be estimated for the brown dwarf candidates using the DM97 mass estimates and radii derived from the luminosity and effective temperature. Values range from $\log (\mathrm{g})=3.0$ to 4.5 in cgs units with a median value of 3.3. These estimates assume a temperature scale, intrinsic colors, and bolometric corrections derived from dwarf standards and only increase under the assumption of giant properties. Therefore, the surface gravities of our objects more closely resemble dwarfs than giants, implying that our use of a dwarf temperature scale has led to an underestimation of the temperature for M6-M9 stars of $<250 \mathrm{~K}$. When taken along with the results of the two sets of models, we conclude that at least 9 of the candidates can be identified as true brown dwarfs.

\section{References}

Aspin, C., Sandell, G., \& Russell, A. 1994, A\&A, 106, 165

Baraffe, I., Chabrier, G., Allard, F., Hauschildt, P. 1998, A\&A, 337, 403

Belikov, A., Kharchenko, N., Piskunov, A., Schilbach, E., \& Scholz, R.-D. 2002, A\&A, 387, 117

Burrows, A., Marley, M., Hubbard, W. B., Lunine, J. L., Guillot, T., Saumon, D., Freedman, R., Sudarsky, D., \& Sharp, C. 1997, ApJ, 491, 856

Cushing, M., Tokunaga, A., \& Kobayashi, N. 2000, AJ, 119, 3019

D'Antona, F. \& Mazzitelli, I. 1997, in Cool Stars in Clusters and Associations, eds. R. Pallavicini \& G. Micela, Mem. S. A. It., 68, n.4. (DM97)

D'Antona, F. \& Mazzitelli, I. 1998, priv. comm.

de Zeeuw, P., Hoogerwerf, R., \& de Bruijne, J. 1999, AJ, 117, 354

Fleming, T., Schmitt, J., \& Giampapa, M. 1995, ApJ, 450, 401

Getman, K., Feigelson, E., Townsley, L., Bally, J., Lada, C., \& Reipurth, B. 2002, ApJ, in press

Herbig, G. 1974, Lick Obs. Bull. No. 658

Itoh, Y., Tamura, M., \& Tokunaga, A. 2002, PASJ, in press

Jones, H., Longmore, A., Jameson, R. \& Mountain, C. 1994, MNRAS, 267, 413

Jones, H., Longmore, A., Allard, F., Hauschildt, P., Miller, S., \& Tennyson, J. 1995, MNRAS, 277, 767

Lada, C., Alves, J., \& Lada, E. 1996, AJ, 111, 1964

Loren, R. 1976, ApJ, 209, 466

Lucas, P., Roche, P., Allard, F. \& Hauschildt, P. 2001, MNRAS, 326, 695

Najita, J., Tiede, G., \& Carr, J. 2000, ApJ, 541, 977

Sargent, A. 1979, ApJ, 233, 163

Strom, S., Vrba, F., \& Strom, K. 1976, AJ, 81, 314

Wilking, B., Greene, T., \& Meyer, M. 1999, AJ, 117, 469 (WGM99)

Wilking, B., Meyer, M., Greene, T., Mikhail, A., \& Carlson, G. 2002, in prep. 


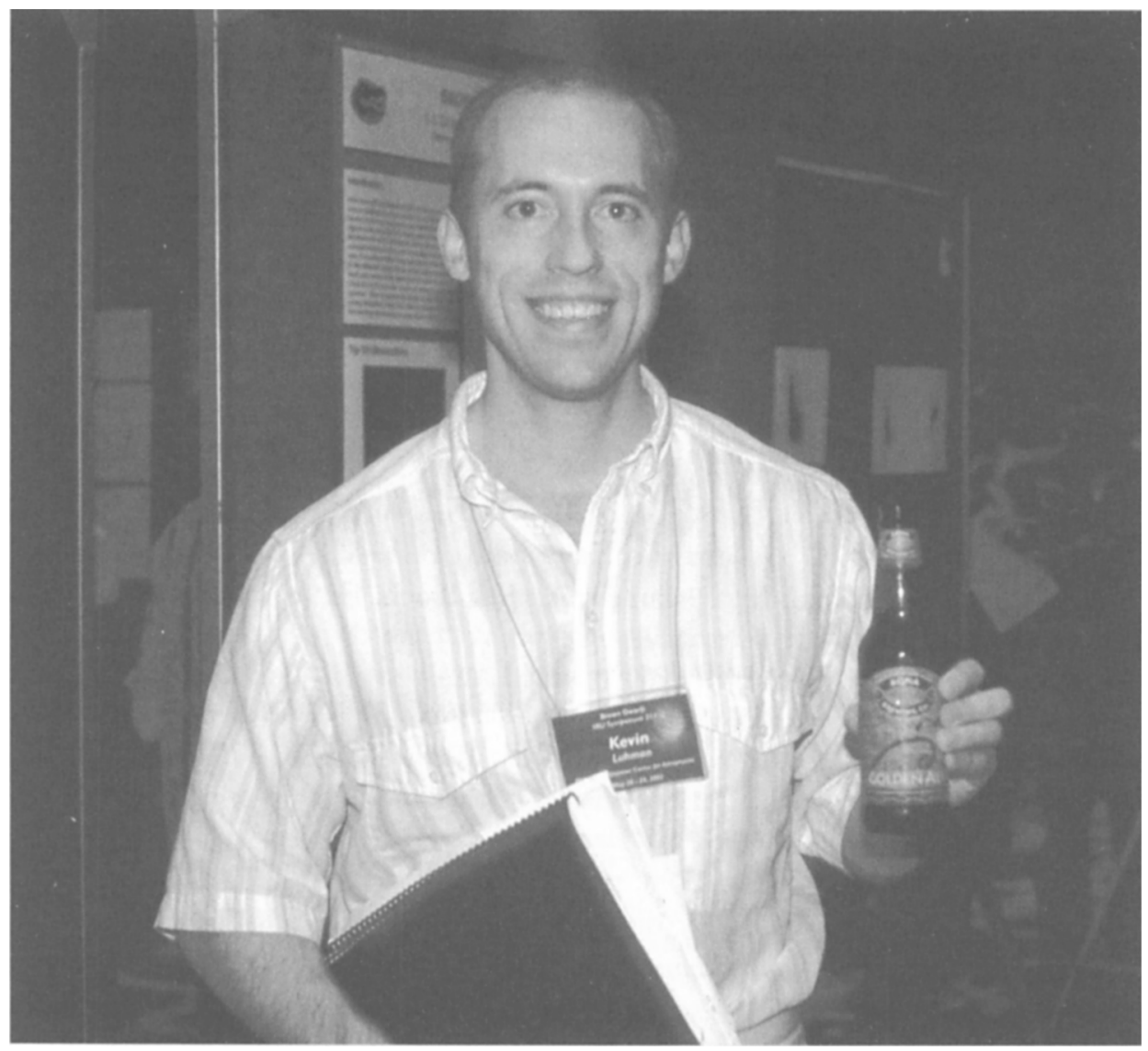

Kevin Luhman 